\title{
BMJ Open Examination of CIs in health and medical journals from 1976 to 2019: an observational study
}

\author{
Adrian Gerard Barnett (D , ${ }^{1}$ Jonathan D Wren ${ }^{2,3}$
}

To cite: Barnett AG, Wren JD. Examination of $\mathrm{Cls}$ in health and medical journals from 1976 to 2019: an observational study. BMJ Open 2019;9:e032506. doi:10.1136/ bmjopen-2019-032506

- Prepublication history and additional material for this paper are available online. To view these files, please visit the journal online (http://dx.doi. org/10.1136/bmjopen-2019032506).

Received 21 June 2019 Revised 04 November 2019 Accepted 06 November 2019

D Check for updates

(c) Author(s) (or their employer(s)) 2019. Re-use permitted under CC BY. Published by BMJ.

${ }^{1}$ Institute of Health and Biomedical Innovation, Queensland University of Technology, Kelvin Grove, Queensland, Australia ${ }^{2}$ Arthritis and Clinical Immunology Research Program, Division of Genomics and Data Sciences, Oklahoma Medical Research Foundation, Oklahoma City, Oklahoma, USA

${ }^{3}$ Department of Biochemistry and Molecular Biology, University of Oklahoma Health Sciences Center, Oklahoma City, Oklahoma, USA

Correspondence to Dr Adrian Gerard Barnett; a.barnett@qut.edu.au

\begin{abstract}
Objectives Previous research has shown clear biases in the distribution of published $p$ values, with an excess below the 0.05 threshold due to a combination of $p$ hacking and publication bias. We aimed to examine the bias for statistical significance using published confidence intervals.

Design Observational study.

Setting Papers published in Medline since 1976.

Participants Over 968000 confidence intervals extracted from abstracts and over 350000 intervals extracted from the full-text.

Outcome measures Cumulative distributions of lower and upper confidence interval limits for ratio estimates.

Results We found an excess of statistically significant results with a glut of lower intervals just above one and upper intervals just below 1. These excesses have not improved in recent years. The excesses did not appear in a set of over 100000 confidence intervals that were not subject to $p$-hacking or publication bias.

Conclusions The huge excesses of published confidence intervals that are just below the statistically significant threshold are not statistically plausible. Large improvements in research practice are needed to provide more results that better reflect the truth.
\end{abstract}

\section{INTRODUCTION}

Every health and medical researcher is aware of the importance of statistically significant results, generally meaning a $\mathrm{p}$ value less than 0.05. A study with statistically significant results is easier to publish in a journal ${ }^{1}$ and statistical significance can be mistaken for study validity and importance. ${ }^{2}$ This can lead some researchers to strive for statistically significant results by reanalysing data to get a $\mathrm{p}$ value under 0.05 , known as "p-hacking'. 34 This striving for statistical significance is not always overt and can occur due to researchers making seemingly sensible scientific decisions. ${ }^{5}$ It can also motivate researchers to carefully vet which hypotheses they pursue, selecting those they deem likely to yield significant results. For example, around 75\% of scientific effort goes towards the $10 \%$ of genes already best characterised, suggesting widespread risk-aversion. ${ }^{6}$

\section{Strengths and limitations of this study}

This is the first study to examine the bias towards statistical significance using confidence intervals instead of $p$ values.

- We used a very large sample of confidence intervals from both abstracts and full-texts.

- We used a simple graphical summary and did not develop a new test to detect $p$-hacking or examine differences in papers around the statistically significant threshold.

- We have taken a broad look at the combined evidence and have not examined individual studies to show that they were biased.

Journals are also somewhat responsible for the bias towards statistically significant results. Journals depend on readership, and journal editors know that readers tend to be more interested in striking results.

The combined 'significance seeking' behaviour of researchers and journals has created a statistically implausible excess of published $\mathrm{p}$ values just below the 0.05 threshold. ${ }^{178}$ This warped evidence undermines the purpose of evidence-based practice, as 'negative' studies too often go unpublished and some published effect estimates are too large because p-hacking inflates effect sizes (by conditioning on statistically significant results). This biased evidence can harm the public's health when policy decisions are made using incomplete or overly optimistic evidence.

Examining published $\mathrm{p}$ values using the 'p-curve' distribution can be used to indicate that p-hacking has occurred. ${ }^{3}$ In this paper, we show that the same excess occurs in confidence intervals, which are a recommended alternative to $\mathrm{p}$ values for presenting results and have been advocated as a way to avoid 'bright-line' thinking at the $0.05 \mathrm{p}$ value threshold. ${ }^{9-11}$ We therefore take an alternative look at the already illustrated bias for statistical significance. However, we think 
this is worthwhile replication because this bias is a crucial problem for evidence-based medicine. Also using confidence intervals may lead to a wider understanding of the bias because researchers often misunderstand $p$ values. ${ }^{12}$ We also show that the bias has not abated in recent years.

As a reminder, a $p$ value is the probability, under a specified model, of observing a test statistic as or more extreme than that observed in the data. A $95 \%$ confidence interval is a range that should contain the true value on $95 \%$ of occasions if the data generating process could be repeated many times. Most confidence intervals are given as $95 \%$ intervals, which correspond to a $0.05 \mathrm{p}$ value threshold.

\section{METHODS}

We extracted ratio confidence intervals from the abstracts and full-texts of journals using regular expressions; for full details, see Georgescu and Wren. ${ }^{8}$ The text-mining algorithm was designed to recognise the typical ways in which ratio estimates (eg, odds ratios, hazard ratios and risk ratios) and confidence intervals are presented. The results were independently validated using a separate text-mining algorithm developed by the first author (AGB). Random checks were made on approximately 5000 abstracts and 500 full texts, as well as other checks on unusual results, for example, extremely wide confidence intervals. The complete codes and data are available at https://github.com/jdwren/ASEC and https:// github.com/agbarnett/intervals.

We excluded the $0.1 \%$ of lower intervals that were zero, assuming this was an error by the authors. We excluded the $0.7 \%$ of abstract intervals and $0.9 \%$ of full-text intervals where the mean was not within the confidence interval, as this meant either the interval or mean (or both) was incorrect.

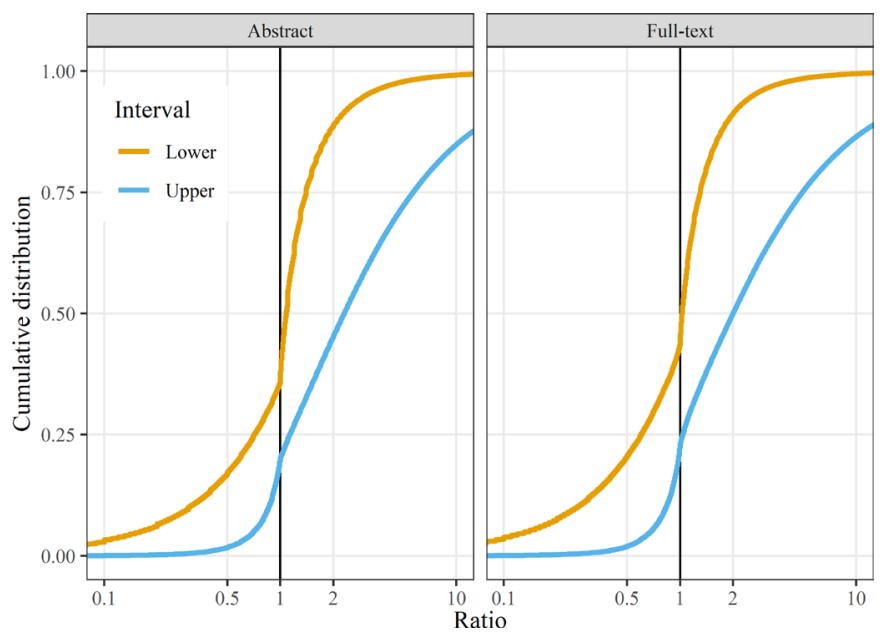

Figure 1 Empirical cumulative distributions for ratio Cls from Medline abstracts and full papers. To be statistically significant, lower intervals need to be above 1 and upper intervals need to be under 1 (vertical lines). The $\mathrm{x}$-axes are restricted to ratios between 0.25 and 4 .
We used a second set of published estimates from health research based on thousands of analyses with no p-hacking or publication bias. ${ }^{13}$ Using four large databases of insurance claims, the study examined all possible pairs of 17 treatments for depression using 22 outcomes. Risks were estimated using survival analysis, giving hazard ratios. Treatment-Outcome pairs were categorised as 'negative' where there was no evidence in the literature of any association. 'Positive' associations were created by simulating an increased risk with a hazard ratio of 1.5. Details are in the paper by Schuemie et al: ${ }^{13}$ the key point for our analysis is that we have a large sample of ratio confidence intervals from Treatment-Outcome pairs that are not subject to any 'significance seeking' by researchers or journals.

\section{Statistical methods}

To summarise the intervals, we plotted the cumulative empirical distribution of the lower and upper confidence interval limits. We used the cumulative distribution because it highlights a change in the distribution, which is our key interest, and it does not require any user-defined tuning parameters. Histograms require the selection of a binwidth and density plots require userdefined smoothing parameters, which are subjective and will create different impressions depending on the choice made. We include histograms in the online supplementary file using a binwidth of 0.1 , but our results focus on the cumulative distributions.

Our hypothesis was that there would be a large change in the intervals near 1 , which is the commonly used null hypothesis of no difference on a ratio scale. A lower confidence interval limit for a ratio estimate that is greater than one would mean a statistically significant result, as would an upper interval limit below 1 . To look for changes over time, we plotted separate cumulative densities in 5-year periods.

We used R V.3.6.0 for all analyses. ${ }^{14}$

\section{Patient and public involvement}

No patients or members of the public were involved in this study.

\section{RESULTS}

Our sample had over 968000 intervals extracted from Medline abstracts from over 5900 journals, and over 350000 intervals extracted from the full-text from over 2700 journals.

For $11 \%$ of intervals, the level of the confidence interval could not be determined, most often because it was not given. Where an interval could be determined, 99.7\% were $95 \%$ confidence intervals, which corresponds to the commonly used $\mathrm{p}$ value threshold of 0.05 .

We found a clear and sudden change in the cumulative distribution of both lower and upper interval limits at the statistically significant threshold of 1 (figure 1). There was a steep increase in the number of lower interval limits 


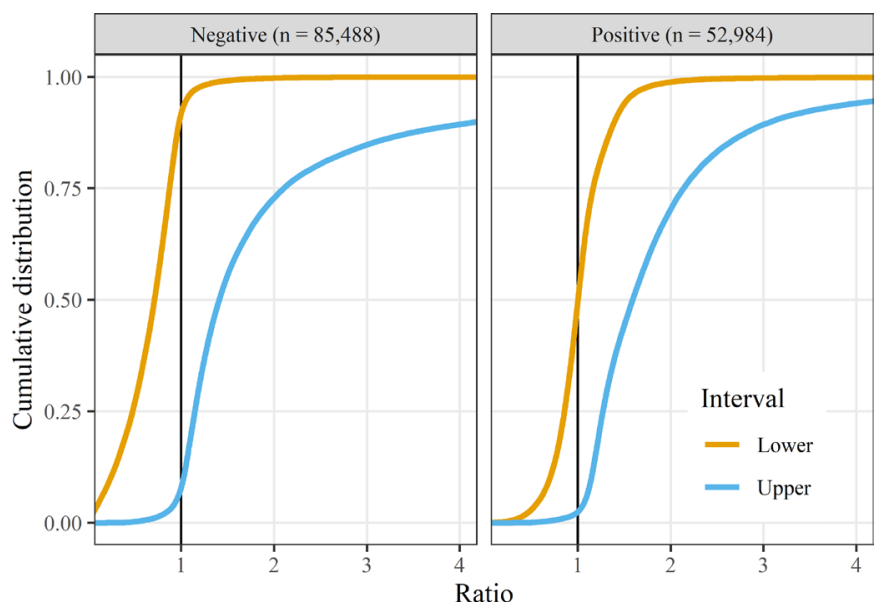

Figure 2 Empirical cumulative distributions for ratio $\mathrm{Cl}$ limits from a sample with no p-hacking or publication bias. 'Negative results were for associations with no known evidence of an association and 'positive' studies were simulated using an increased risk. The $\mathrm{x}$-axes are restricted to ratios between 0.25 and 4 .

just above 1, meaning they were just above the threshold for statistical significance. Similarly, there was a steep increase in the number of upper interval limits just below 1 and so just inside the statistically significant threshold. The discontinuity at 1 appears slightly stronger (less smooth) in the abstract than the full-text and there are noticeably more lower intervals below 1 in the full-text, which suggests that the biases are stronger in the abstract than the full-text.

If there was no p-hacking and publication bias, the cumulative distributions would be smooth S-shapes with no discontinuities, and this is confirmed in figure 2 using the data without these biases.

The bias for statistically significant results in abstracts has persisted over time as shown by the 5 -year plots in figure 3 . The plots also show the reduced use of rounded

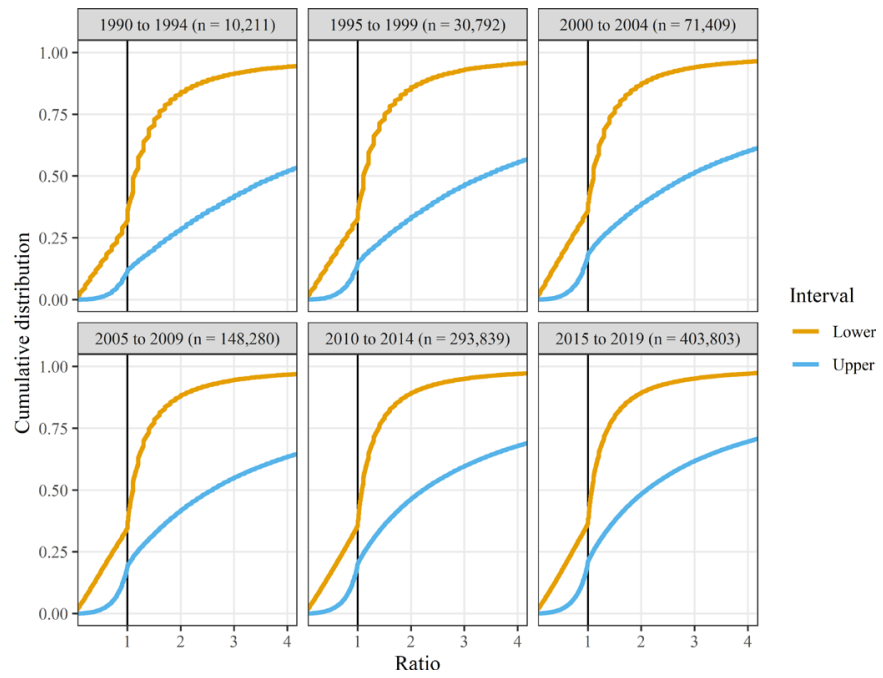

Figure 3 Empirical cumulative distributions for ratio Cls from Medline abstracts in 5-year intervals. The $\mathrm{x}$-axes are restricted to ratios between 0.25 and 4 . intervals over time, as the cumulative densities are smoother in later years.

The online supplementary file contains sensitivity analyses that use wider and narrow ratio axes, show histograms instead of cumulative densities and show plots of the cumulative densities over time for the full-text. All the plots show the same striking changes in the number of intervals near 1 .

\section{DISCUSSION}

The strong bias for statistically significant results is clearly visible in the confidence intervals of a large sample of abstracts and full-texts from health and medical journals and has persisted for decades. This bias undermines the evidence base and impairs good decision making by giving a false picture of the effectiveness of treatments and procedures. Such biased evidence can harm patients and the public when it gets translated into policy and practice.

Confidence intervals have been promoted as a way of avoiding the simplistic bright-line thinking that often accompanies $p$ values. However, our results show that even though people are frequently reporting confidence intervals, many are still focusing on statistical significance. A push towards the greater use of confidence intervals in journals in place of $p$ values may therefore not solve the ongoing problem of bright-line thinking. ${ }^{15}$

Large improvements in research practice are needed to improve the quality of evidence. Study results should be presented and published regardless of their statistical significance. This could be achieved by a wider uptake of study preregistration and analysis plans, ${ }^{16}$ which reduces the potential for researchers to change their hypothesis or primary outcome after seeing the data.

Statistical significance should no longer be used as a tool to screen what results are published and the evidence base would be in a better state if significance were given far less prominence. ${ }^{17}$ The prominence given to statistical significance is ironic given the widespread misunderstandings of its true meaning. ${ }^{2} 12$

\section{Limitations}

We did not aim to develop a new statistical test for p-hacking or numerically compare the extent of p-hacking in abstracts versus full-texts or our sample of the published literature versus the sample with no p-hacking. Instead our aim was to present some simple plots that highlight the extent of the problem.

We included all confidence intervals, but we anticipate the biases would be even more striking if we were able to restrict the sample to the primary outcomes of interest. ${ }^{18}$ This is because the statistical significance of the primary outcomes dictate the 'success' or 'failure' of the study, and less high-profile comparisons (eg, between groups at baseline) are less likely to be p-hacked.

We have taken a broad look at the combined evidence and have not examined individual studies to show that 
they were biased, nor did we estimate the size of the bias. Instead, we aimed to show the extent of the bias across the literature using a simple graphical method. To our knowledge, this is the first paper to examine the problem using confidence intervals and uses one of the largest sample sizes including results from both abstracts and full-texts. For us, the graphs are a warning of the urgent need for action to improve research practices.

\section{Twitter Adrian Gerard Barnett @aidybarnett}

Contributors AGB and JW conceived the idea. JW wrote the programs to extract the data. AGB wrote the analyses code and the first draft of the paper with comments from JW. AGB is responsible for the overall content and is the guarantor.

Funding AGB was supported by Queensland University of Technology and the National Health and Medical Research Council grant number APP1117784.

Competing interests None declared.

Patient consent for publication Not required.

Provenance and peer review Not commissioned; externally peer reviewed.

Data availability statement Data are available in a public, open access repository.

Open access This is an open access article distributed in accordance with the Creative Commons Attribution 4.0 Unported (CC BY 4.0) license, which permits others to copy, redistribute, remix, transform and build upon this work for any purpose, provided the original work is properly cited, a link to the licence is given, and indication of whether changes were made. See: https://creativecommons.org/ licenses/by/4.0/.

ORCID iD

Adrian Gerard Barnett http://orcid.org/0000-0001-6339-0374

\section{REFERENCES}

1 Emerson GB, Warme WJ, Wolf FM, et al. Testing for the presence of positive-outcome bias in peer review. Arch Intern Med 2010;170.
2 Ziliak S, McCloskey DN. The cult of statistical significance: how the standard error costs us jobs, justice, and lives. University of Michigan Press, 2008.

3 Simonsohn U, Nelson LD, Simmons JP. $p$-Curve and Effect Size. Perspectives on Psychological Science 2014;9:666-81.

4 Head ML, Holman L, Lanfear R, et al. The extent and consequences of p-hacking in science. PLoS Biol 2015;13:e1002106-15.

5 Gelman A, Loken E. The garden of forking paths: Why multiple comparisons can be a problem, even when there is no "fishing expedition" or "p-hacking" and the research hypothesis was posited ahead of time, 2013. Available: http://www.stat.columbia.edu/ gelman/research/unpublished/p_hacking.pdf

6 Edwards AM, Isserlin R, Bader GD, et al. Too many roads not taken. Nature 2011;470:163-5.

7 Chavalarias D, Wallach JD, Li AHT, et al. Evolution of reporting $P$ values in the biomedical literature, 1990-2015. JAMA 2016;315:1141-8.

8 Georgescu C, Wren JD. Algorithmic identification of discrepancies between published ratios and their reported confidence intervals and P-values. Bioinformatics 2018;34:1758-66.

9 Ranstam J. Why the P-value culture is bad and confidence intervals a better alternative. Osteoarthritis and Cartilage 2012;20:805-8.

10 Wasserstein RL, Lazar NA. The ASA Statement on $p$-Values: Context, Process, and Purpose. Am Stat 2016;70:129-33.

11 Lee DK. Alternatives to $P$ value: confidence interval and effect size. Korean J Anesthesiol 2016;69:555.

12 Goodman S. A dirty dozen: twelve P-Value misconceptions. Semin Hematol 2008;45:135-40.

13 Schuemie MJ, Ryan PB, Hripcsak G, et al. Improving reproducibility by using high-throughput observational studies with empirical calibration. Philosophical Transactions of the Royal Society A: Mathematical, Physical and Engineering Sciences 2018;376.

$14 \mathrm{R}$ Core Team. $R$ : a language and environment for statistical computing. Vienna, Austria: R Foundation for Statistical Computing, 2018.

15 Fidler F, Thomason N, Cumming G, et al. Editors Can Lead Researchers to Confidence Intervals, but Can't Make Them Think. Psychol Sci 2004;15:119-26.

16 Kaplan RM, Irvin VL. Likelihood of null effects of large NHLBI clinical trials has increased over time. PLoS One 2015;10:e0132382.

17 McShane BB, Gal D. Statistical significance and the Dichotomization of evidence. J Am Stat Assoc 2017;112:885-95.

18 Simonsohn U. [41] falsely reassuring: Analyses of ALL p-values, 2015. Available: http://datacolada.org/41 